\title{
Dynamic angle selection in X-ray computed tomography
}

\author{
Andrei Dabravolski ${ }^{\mathrm{a}, *}$, Kees Joost Batenburg ${ }^{\mathrm{a}, \mathrm{b}}$, Jan Sijbers ${ }^{\mathrm{a}}$ \\ a iMinds-Vision Lab, University of Antwerp, Universiteitsplein 1, 2610 Wilrijk, Belgium \\ ${ }^{\mathrm{b}}$ Centrum Wiskunde E Informatica (CWI), Science Park 123, 1098 XG Amsterdam, The Netherlands
}

\section{A R T I C L E I N F O}

Article history:

Received 7 June 2013

Received in revised form 30 July 2013

Accepted 28 August 2013

Available online 5 February 2014

\section{Keywords:}

Angle selection

Computed tomography

Dynamic imaging

Information gain

\begin{abstract}
A B S T R A C T
In X-ray tomography, a number of radiographs (projections) are recorded from which a tomogram is then reconstructed. Conventionally, these projections are acquired equiangularly, resulting in an unbiased sampling of the Radon space. However, especially in case when only a limited number of projections can be acquired, the selection of the angles has a large impact on the quality of the reconstructed image. In this paper, a dynamic algorithm is proposed, in which new projection angles are selected by maximizing the information gain about the object, given the set of possible new angles. Experiments show that this approach can select projection angles for which the accuracy of the reconstructed image is significantly higher compared to the standard angle selections schemes.
\end{abstract}

(c) 2014 Elsevier B.V. All rights reserved.

\section{Introduction}

Tomography has applications ranging from 3D imaging of nano-materials by electron microscopy to the reconstruction of accretion disks from astronomical observations. In many of these applications, it is highly desirable to reduce the number of projections taken, or it is even impossible to acquire many projections. In image-guided radiotherapy, for example, a patient is being imaged for several times posing a serious radiation safety concern [1]. In astro-tomography, only a few satellites are capable of imaging the corona of the sun, leading to long acquisition times. In electron tomography, the electron beam gradually damages the object, also imposing a restriction on the number of projections that can be acquired [2].

When an image is being reconstructed from a small number of projections, the angles from which these projections will be acquired will significantly influence the reconstruction quality. In [3], it was shown that the quality of the reconstructions can be highly dependent on the projection angles in binary tomography. In that paper, an algorithm was proposed for identifying optimal projection angles based on a blueprint image known to be similar to the scanned object, which can be readily applied in the field of non-destructive testing. For the more general case of grey scale tomography, a framework was proposed in [4], which allows to optimize the set of projection angles based on certain prior knowledge about the object. In [5], an algorithm was proposed to select new projection angles based on the quantification of the projection

\footnotetext{
* Corresponding author. Tel.: +32 32652840; fax: +32 32652245.

E-mail addresses: andrei.dabravolski@uantwerpen.be (A. Dabravolski), joost. batenburg@uantwerpen.be (K.J. Batenburg), jan.sijbers@uantwerpen.be (J. Sijbers).
}

information content using an entropy-like function of the already acquired projections. For tomography of elliptical objects, a genetic algorithm was proposed [6], which exploits the preferential direction characteristic of the objects and uses reconstructions from available projections to select the next projection directions. In [7], a new strategy was recently proposed for angle selection in binary tomography, which is based on the concept of information gain from adding a particular projection angle to the set of projection directions and does not require specifying prior knowledge about the object.

In the present paper, the dynamic angle selection strategy for binary object scanning is adapted for use in grey scale tomography. It is a dynamic algorithm, which selects a new angle based on the currently available projection data and incorporates two major concepts: (1) sampling of the set of images that are consistent with the already acquired projection data and (2) determining the amount of information that can be gained by acquiring a projection from a particular angle.

The structure of this paper is as follows. In Section 2 our approach is explained. Section 3 describes experiment setups and presents obtained results. The approach is discussed in Section 4. Finally, conclusions are drawn in Section 5.

\section{Method}

\subsection{Information gain}

The idea of the proposed angle selection algorithm is to select a new projection direction in such a way that the newly obtained projection will contain as much information about the object as possible. As a measure of information, a concept of information 
gain is used, which is based on the diameter of the set of solutions that are consistent with already obtained projections [7].

Let $\Theta=\left\{\theta_{1}, \ldots, \theta_{d}\right\}$ be the current set of $d$ angles, for which projection data $p^{\Theta}=W^{\Theta} v$ of the unknown image $v \in[0,1]^{n}$ have already been measured, where $n$ is the number of pixels in the image and $W^{\Theta}$ is the projection matrix corresponding to $\Theta$. Note that if the assumption on the range of the grey values of the unknown image is not satisfied in practice, a preprocessing step is needed to make the assumption valid, which is discussed in Section 4. Let $S_{W^{\Theta}}\left(p^{\Theta}\right)=\left\{x \in[0,1]^{n}: W^{\Theta} x=p^{\Theta}\right\}$ be the set of all solutions that are consistent with the projection data $p^{\Theta}$. Then, the information gain for any image $x$ and set of angles $\Theta$ yielded by taking a projection from angle $\theta$ is defined by

$G(x, \Theta, \theta)=\operatorname{diam}\left(S_{W^{\Theta}}\left(W^{\Theta} x\right)\right)-\operatorname{diam}\left(S_{W^{\Theta \cup\{\theta\}}}\left(W^{\Theta \cup\{\theta\}} x\right)\right)$,

where $\operatorname{diam}(V)=\max \left\{\|x-y\|_{2} \mid x, y \in V\right\}$ for any $V \subset[0,1]^{n}$. This defines the information gain as the difference of the diameters of the sets of all images having the same projections as $x$. Having defined the mean information gain of a set of images $V \subset[0,1]^{n}$ as

$G(V, \Theta, \theta)=\sqrt{\frac{\int_{V} G(x, \Theta, \theta)^{2} \mathrm{~d} x}{\int_{V} \mathrm{~d} x}}$,

the next projection angle can be found as

$\theta_{d+1}=\arg \max _{\theta \in[0, \pi)} G\left(S_{W^{\Theta}}\left(p^{\Theta}\right), \Theta, \theta\right)$.

In practice, the integrals in Eq. (2) have to be approximated. In the present paper, three approximation steps are proposed. Firstly, the diameter of the solution set in Eq. (1) is substituted with its upper bound which was proposed in $[8,9]$ for binary solutions and which also holds for the solutions belonging to $[0,1]^{n}$. Secondly, integration in Eq. (2) is replaced by a summation over a set of surrogate solutions, which represent the true set $S_{W^{\Theta}}\left(p^{\Theta}\right)$. Finally, the continuous domain of the candidate angle $\theta_{d+1}$ is substituted with a finite set of candidate angles, updated every time a new angle is chosen. These steps are explained in detail in the following sections.

\subsection{Upper bound for the diameter of the solution set}

The upper bound for the diameter used in Eq. (1) is based on the concept of the central reconstruction $x^{*}$, which is the shortest solution in $S_{W^{\Theta}}\left(p^{\Theta}\right)$, in the Euclidean sense. This reconstruction can be computed using the Conjugate Gradient Least Squares (CGLS) method, an iterative Krylov subspace method ([10]). Define the

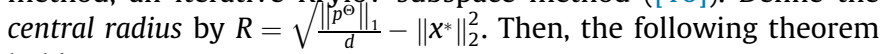
holds:

Theorem. Let $x, y \in S_{W^{\Theta}}\left(p^{\Theta}\right)$. Then $\|x-y\|_{2} \leq 2 R$.

The proof of this theorem can be found in Appendix A.

\subsection{Surrogate solutions}

In order to evaluate the mean information gain defined by Eq. (2), the fraction under the square root is replaced by the mean information gain over the set of surrogate solutions, which are used as samples representing the true solution set $S_{W^{\Theta}}\left(p^{\Theta}\right)$. A surrogate solution is calculated from a template image, a randomly generated member of a given parameterized family of images. This template image is then used as a starting point for the Simultaneous Iterative Reconstruction Technique (SIRT) [11] that computes the surrogate solution consistent with already obtained projection data $p^{\Theta}$. As the system $W^{\Theta} x=p^{\Theta}$ is severely underdetermined, the surrogate solution partly retains the features present in the template image. Hence, allowing sufficient variation within the set of template images results in variation of the surrogate solutions obtained and allows to control the approximation of the true solution set $S_{W^{\Theta}}\left(p^{\Theta}\right)$.

\subsection{Candidate angles}

An adaptive approach is proposed to modify the set of angles being considered at the subsequent angle selection steps. Let $A_{d+1}=\left\{\alpha_{1}, \alpha_{2}, \ldots, \alpha_{l}\right\}, 0 \leqslant \alpha_{1} \leqslant \alpha_{2} \leqslant \ldots \leqslant \alpha_{l}<\pi$ be the set of candidate angles for selecting the next angle $\theta_{d+1}$. Suppose that $\alpha_{i}$ is the best angle and $\theta_{d+1}:=\alpha_{i}$. Then, let the candidate angle set used for the selection of the angle $\theta_{d+2}$ be defined as $A_{d+2}=\left\{\alpha_{1}, \ldots, \alpha_{i-1}, \frac{\alpha_{i-1}+\alpha_{i}}{2}, \frac{\alpha_{i}+\alpha_{i+1}}{2}, \alpha_{i+1}, \ldots, \alpha_{l}\right\}$. This procedure allows to better sample the candidate angle space near the angles which are likely to reveal more details in the object and still leaves the possibility for choosing completely new directions.

\subsection{Dynamic angle selection algorithm}

Combining all the approximation steps, an algorithm for estimating the mean information gain for a candidate angle can be defined (Algorithm 1). Based on this algorithm, the proposed angle selection approach iterates over the set of candidate angles and chooses the angle yielding the highest mean information gain.

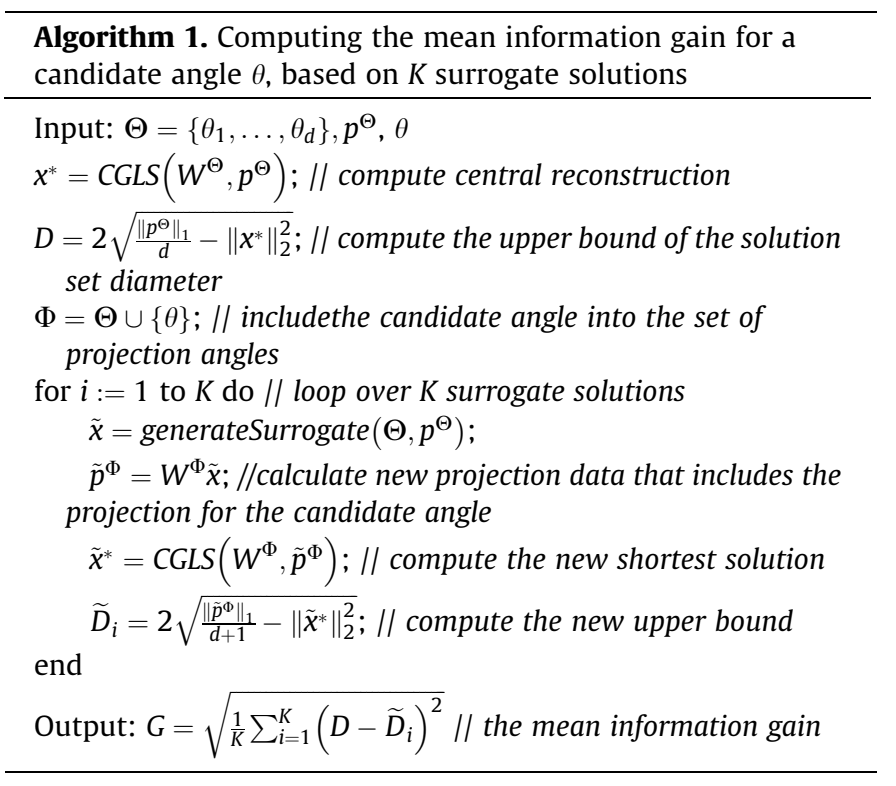

\section{Experiments}

Simulation experiments were run to assess the ability of the proposed algorithm to select favourable projection angles. The size of the phantoms was limited to $128 \times 128$ pixels due to the computational complexity of the approach. A quantitative evaluation

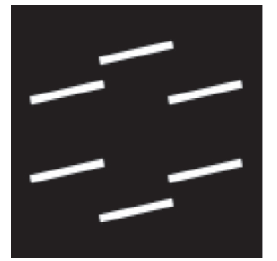

(a)

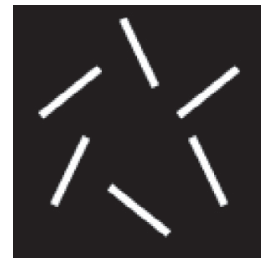

(b)
Fig. 1. Examples of the phantoms with one (a) and four (b) orientations, used in the experiments of Section 3.1 . 


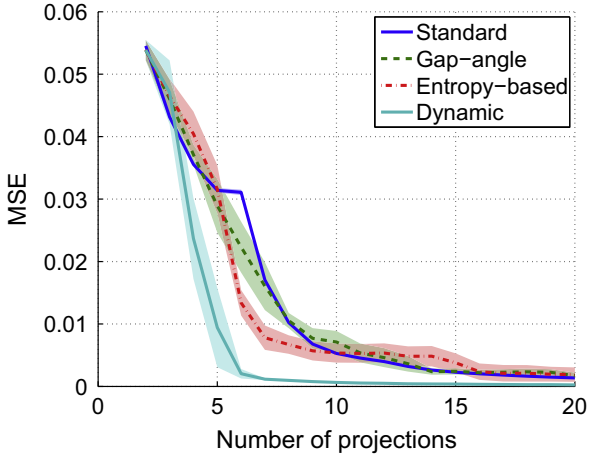

(a)

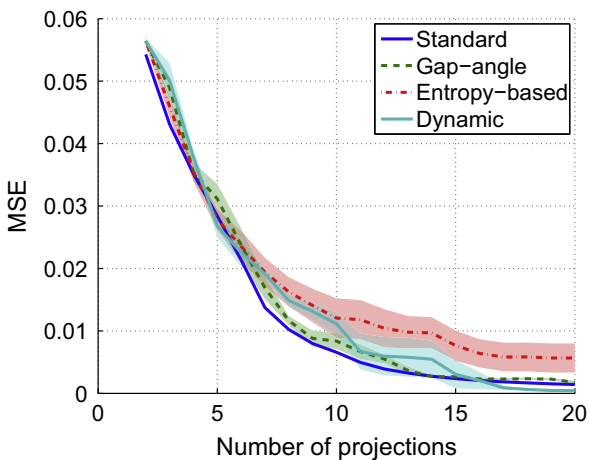

(c)

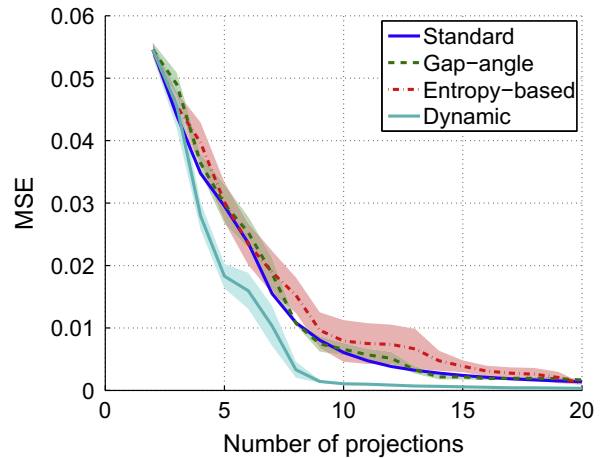

(b)

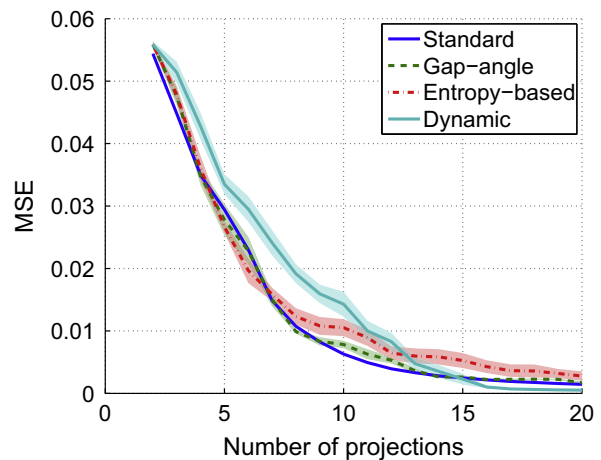

(d)

Fig. 2. MSE as a function of the number of projection angles for phantoms with one to four orientations ((a)-(d), respectively).

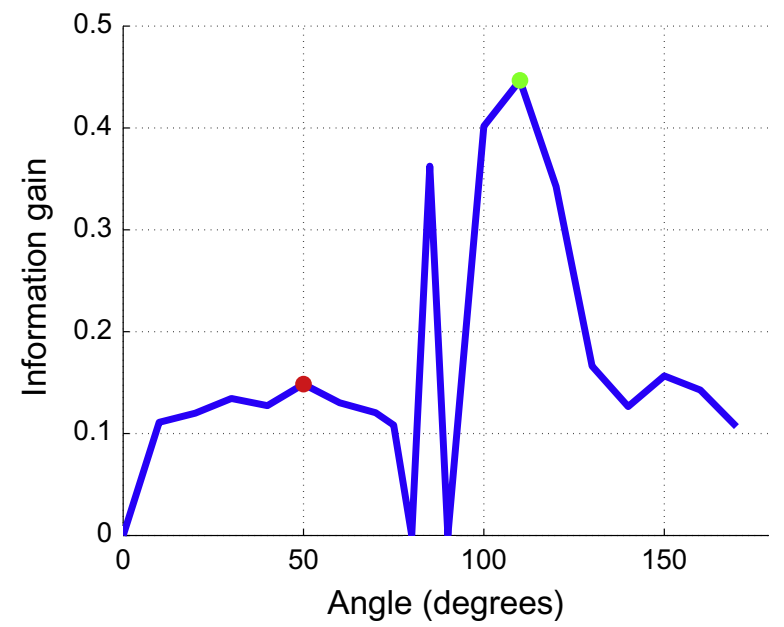

(a)

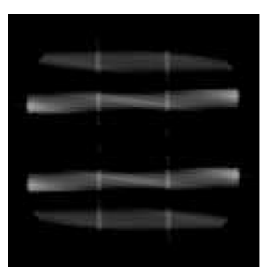

(b)

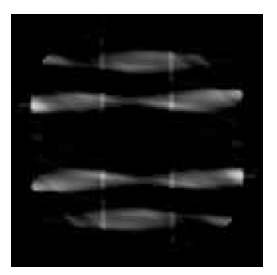

(c)

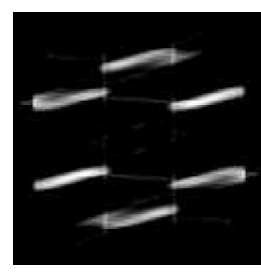

(d)

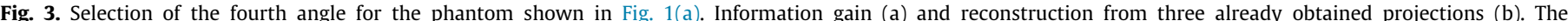

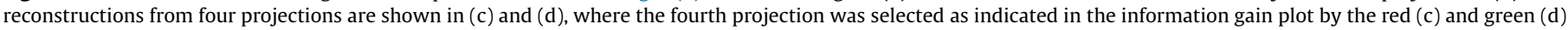
dot, respectively. (For interpretation of the references to color in this figure legend, the reader is referred to the web version of this article.) 
of the proposed algorithm is based on the assumption that a good angle selection scheme will lead to a more accurate reconstruction from fewer angles compared to a reconstruction from angles chosen by a standard selection scheme.

For all presented experiments, the template images used for the generation of the surrogate solutions were created as a superposition of 50 2D Gaussian blobs with randomly chosen orientation and standard deviation along both axes between 3 and 10 pixels. For the selection of each angle, $K=10$ surrogate solutions were generated. Angles with a step of $10^{\circ}$ were chosen for the initial set of candidate angles, which was then modified as described in Section 2.4.

Three angle selection schemes were chosen as antagonists for the proposed algorithm:

- Standard. A widely used strategy, in which angles are selected between $0^{\circ}$ and $180^{\circ}$, with equiangular spacing. Changing the number of angles actually changes the entire set of selected angles, which explains the fluctuations of the numerical results for this strategy.

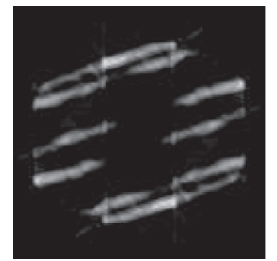

(a)

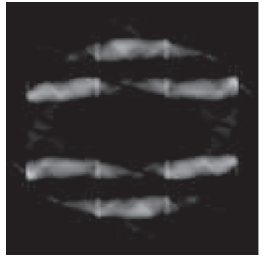

(b)

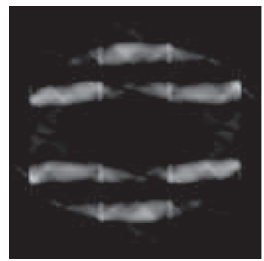

(c)

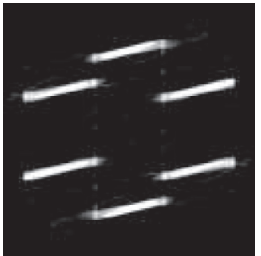

(d)

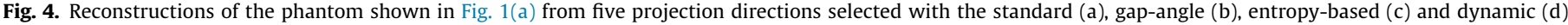
algorithms.

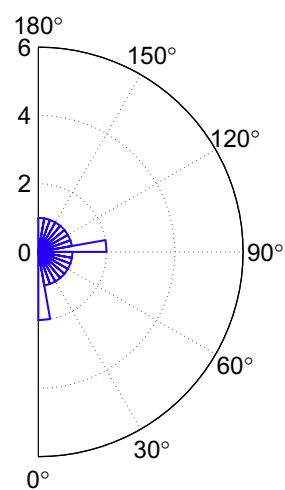

(a)

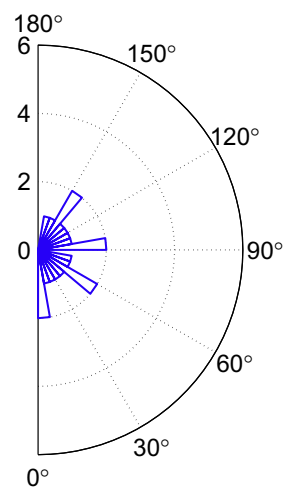

(b)

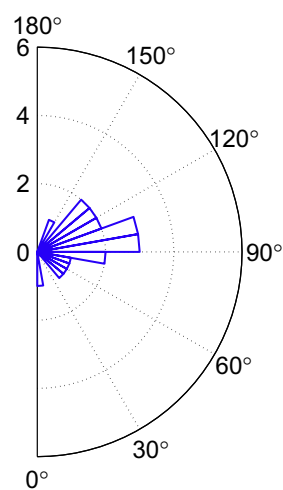

(c)

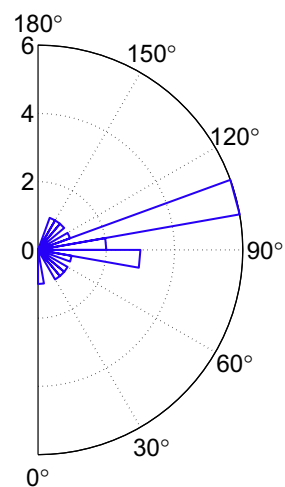

(d)

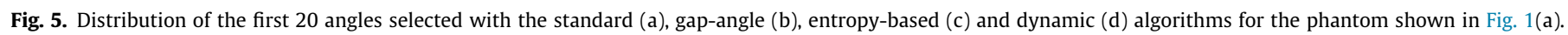

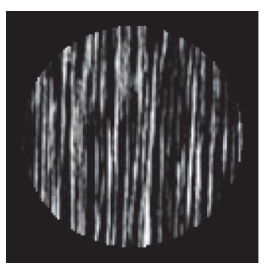

(a)

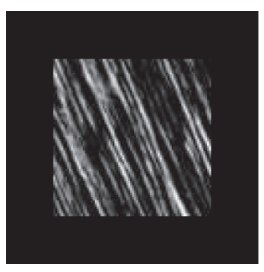

(d)

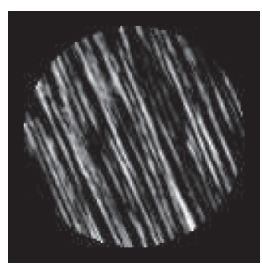

(b)

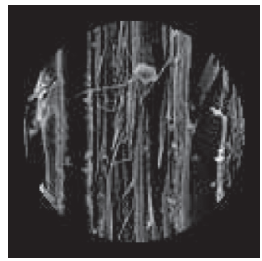

(e)

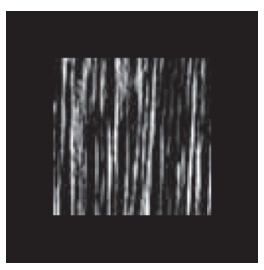

(c)

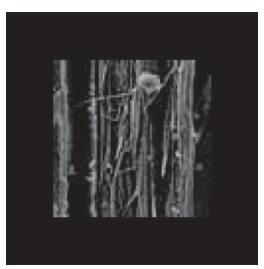

(f)

Fig. 6. Phantoms used in the experiments of Section 3.2. 
- Gap-angle. In the gap-angle scheme, a new angle is selected as the midpoint between the two consecutive angles with the largest angular gap between them. If several pairs of angles have equal gaps, one of them is chosen randomly.

- Entropy-based. In this approach, a new angle is selected based on the "entropy" of the already measured projections. Assuming that $\sum_{i=1}^{m} p_{i}^{\theta}=1$, the entropy $E_{\theta}$ for the projection $p^{\theta}$ is defined as $E_{\theta}=-\sum_{i=1}^{m} p_{i}^{\theta} \log p_{i}^{\theta}$, where $m$ is the number of detector elements and $p_{i}^{\theta} \log p_{i}^{\theta}=0$ for $p_{i}^{\theta}=0$. The next projection is measured between two projections that present the maximum difference in the entropy. For a detailed description and analysis of this approach, the reader is referred to [5].

Nine angle sets were used as a starting point for all algorithms, containing two perpendicular angles and having an angular shift of $10^{\circ}$ with respect to the previous initial angle set, giving 9 starting configurations. For each of the starting configurations, angles were selected with the schemes under consideration and the selected

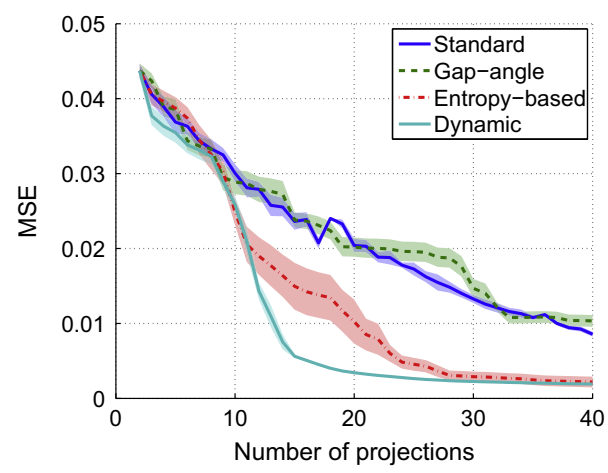

(a)

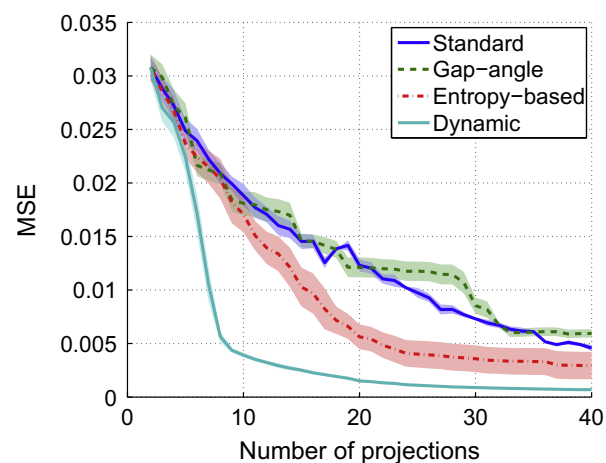

(c)

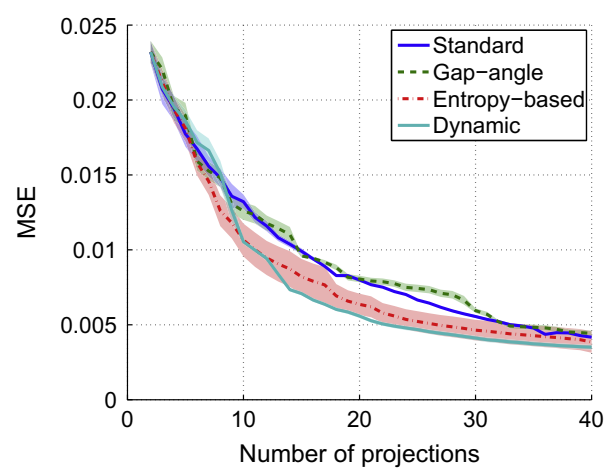

(e) angles were then used to compute reconstructions using 250 iterations of SIRT. The mean values of the mean squared errors (MSEs) of the reconstructions for all starting configurations were then calculated and plotted together with the standard errors (shown as shaded areas in the plots). Two sets of experiments were run, revealing the ability of the algorithms to deal with phantoms having clear direction preferences and to handle more realistic phantoms, which are described in Sections 3.1 and 3.2. For one phantom from Section 3.2 simulations with varying noise levels were also performed, which are described in Section 3.3. All presented experiments were implemented using the ASTRA toolbox [12] where extensive use of GPU acceleration was used [13].

\subsection{Randomly oriented bars}

For the first series of experiments, a set of phantoms was created. A phantom in this set consists of six rectangular bars, each oriented along one of the angles from $\left\{\frac{180^{\circ} i}{7}, i=1, \ldots, 6\right\}$ with

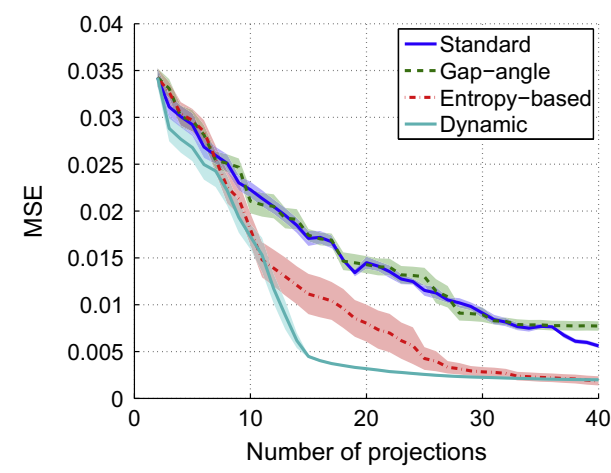

(b)

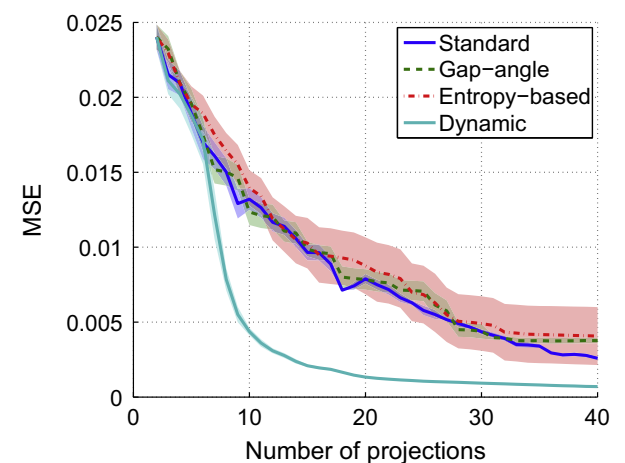

(d)

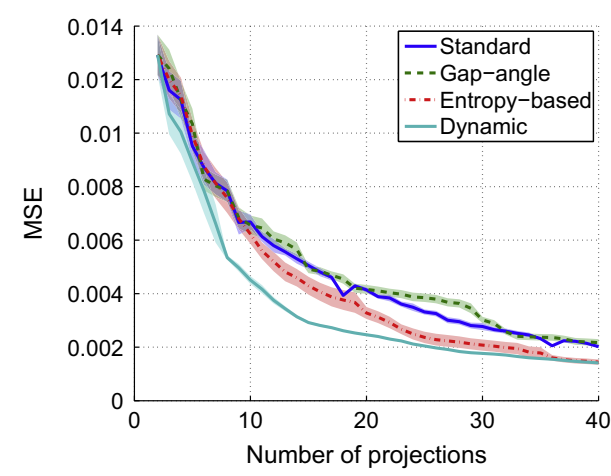

(f)

Fig. 7. MSE as a function of the number of projection angles for Fig. 6(a)-(f). 
respect to the vertical edge of the phantom. The number of different bar-edge angles in the phantom defines the orientation of the phantom. Thus, in the phantom with one orientation, the direction of the bars is the same. In the phantom with six orientations, the orientation of each bar is randomly chosen from the set $\left\{\frac{180^{\circ} i}{7}, i=1, \ldots, 6\right\}$. For the number of orientations from one to four, six phantoms were created with randomly chosen bar-edge angles, giving 24 phantoms in total. Examples of the phantoms with one and four orientations are shown in Fig. 1(a) and (b), respectively. Fig. 2(a)-(d) present average MSEs for the phantoms with equal number of orientations. These plots suggest that the proposed approach has a clear advantage over the other three strategies for the phantoms with one or two orientations and shows comparable or worse results for phantoms with a larger number of orientations, showing strong dependency on the number of orientations in the phantom, whereas the performance of its antagonists demonstrates little to no such dependency.

To illustrate each step of the algorithm, the selection of one angle is considered in detail for the phantom shown in Fig. 1(a). Projections from three angles $\Theta=\left\{0^{\circ}, 80^{\circ}, 90^{\circ}\right\}$ are already available and the corresponding reconstruction is shown in Fig. 3(b). Fig. 3(a) presents the average information gain. Note that zeros in this plot correspond to the already acquired projections. Fig. 3(c) and (d) show the possible reconstructions for cases when angles $50^{\circ}$ and $110^{\circ}$ are chosen as the fourth projection angle. Examples of the reconstructions from five angles yielded by the considered approaches are presented in Fig. 4. Fig. 5 depicts the distribution of 20 angles selected with each algorithm. This example illustrates the correspondence between the average information gain and the reconstruction quality, confirms the ability of the proposed approach to select projection angles according to the directions presented in the object and to yield more accurate reconstructions compared to the standard angle selection schemes.

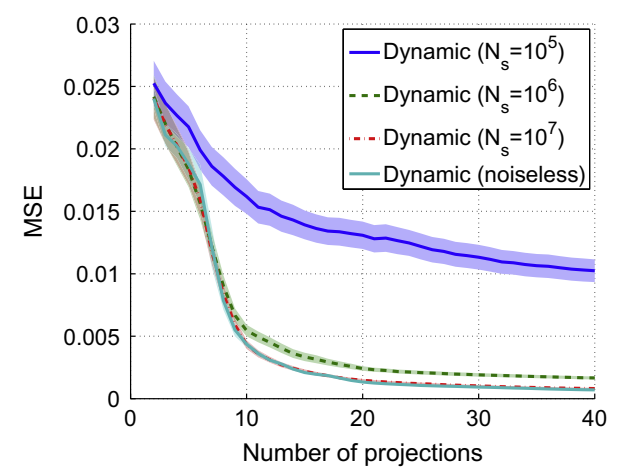

(a)

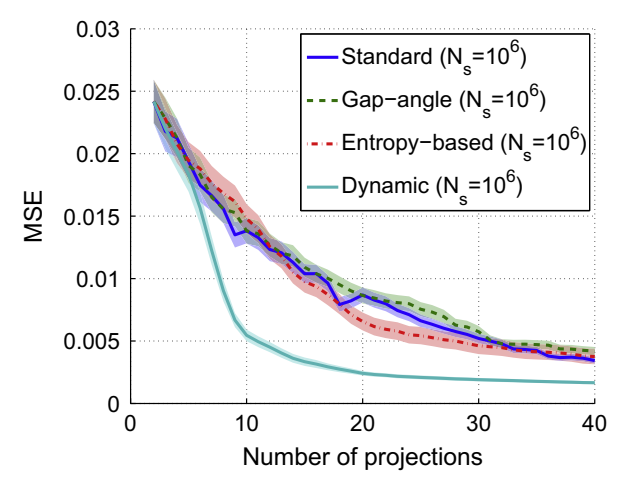

(c)

\subsection{Wood phantoms: noiseless simulations}

For the second series of the experiments, six phantoms (Fig. 6) were created from micro-CT (Fig. 6(a)-(d)) and scanning electron microscope (Fig. 6(e) and 6(f)) images of wood samples. Although these phantoms have clear preferential directions, they are far more complex compared to the bar phantoms described in Section 3.1. The phantoms include fine structures with (cfr. Fig. 6(a)-(d)) or without (cfr. Fig. 6(e) and 6(f)) preferential directions and have two different shapes which either have or do not have preferential directions. Fig. 6(b) and (d) were obtained by rotating Fig. 6(a) and (c), respectively. However, as the phantoms were defined on a pixel grid, some interpolation occurred during the rotation, resulting in minor differences in the results for the proposed algorithm compared to the results for the non-rotated phantoms. Fig. 7 shows the MSEs for Fig. 6(a)-(f), which demonstrate the ability of the proposed approach to yield projection angle sets resulting in more accurate reconstructions from fewer projections. The results for Fig. 6(b) and (d) suggest that the dynamic algorithm accurately reconstructs the rotated objects as well as the unaltered objects. In general, the dynamic angle selection algorithm outperforms the other methods with respect to reconstruction quality. Note that the shape of the phantoms also plays a significant role in the performance of the proposed algorithm, allowing it to reduce the MSE faster as a function of the number of projections for the phantoms that have a square shape compared to circular phantoms.

\subsection{Wood phantom: simulations with noise}

To evaluate the proposed approach in more realistic situations, the experiments shown in Section 3.2 were extended with noise simulations as follows. Poisson noise was simulated in the projec-

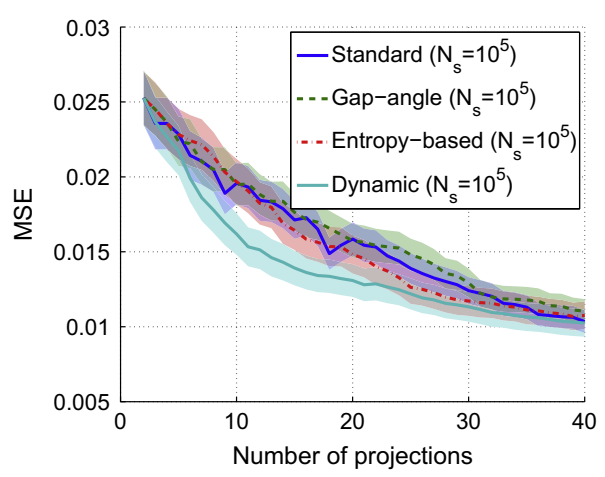

(b)

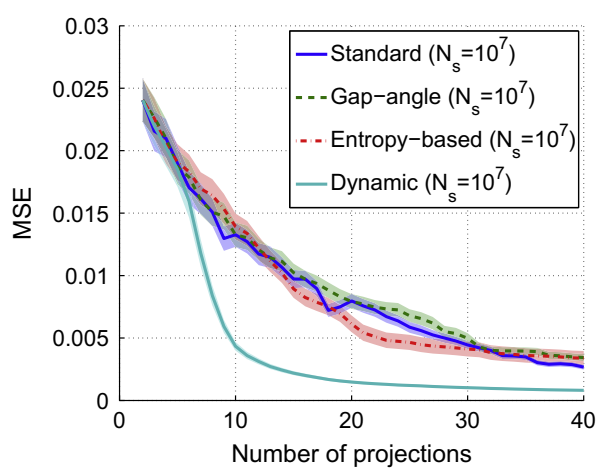

(d)

Fig. 8. MSE as a function of the number of projection angles for the simulations with different noise levels for the proposed algorithm (a) and for all considered algorithms (b-d) for Fig. 6(d). 
tion data based on a number of counts $N_{s}$ for the phantom shown in Fig. 6(d) from Section 3.2. The values $N_{s}=10^{5}, N_{s}=10^{6}$ and $N_{s}=10^{7}$ were used to simulate the different noise levels [14]. The number of SIRT iterations was 250. The mean values of the mean squared errors (MSEs) of the reconstructions as a function of the number of projections were then calculated and plotted together with the standard errors (shown as shaded areas in the plots) in Fig. 8. These results suggest that the proposed algorithm is robust against noise and can provide projection angle sets leading to more accurate reconstructions in the presence of noise. Improvement in the reconstruction quality for the proposed algorithm decreases compared to the standard angle selection schemes as the level of noise increases, yet allowing the dynamic approach to outperform other angle selection strategies for the phantom shown in Fig. 6(d) even in the case of the highest noise level considered.

\section{Discussion}

The proposed dynamic angle selection algorithm selects projection angles based on the already measured projection data by maximizing the information gain. The algorithm does not use any prior knowledge about an object. The only assumption made is that pixel values fall into the range of $[0,1]$. However, even if this assumption is not satisfied, an upper bound for a pixel value can be calculated based on a pixel size and attenuation of the densest material possible (providing that X-rays are not entirely absorbed by the object). The projection data can then be divided by this bound, resulting in the corresponding scaling of the reconstructed image and the above mentioned assumption being satisfied.

Experiments show that the proposed approach can produce angle sets that provide more accurate reconstructions compared to the conventional angle selection strategies or reconstructions of comparable quality from smaller number of projections. The biggest improvement in reconstruction quality is achieved for objects with a few preferential directions in the interior and shape. Intuitively, imaging quality for this type of objects strongly depends on a small set of selected angles, whereas a relatively large number of projections assures high reconstruction quality for any reasonable angle set. In contrast, objects with a high number of preferential directions (or without any preferences) can hardly be reconstructed with acceptable quality from a small number of projections, and the influence of the angle choice for a large number of projection angles decreases (again, for reasonable choices).

Because of the high computational complexity of the method, the current algorithm is not feasible for large image sizes. Possible ways to reduce the computational requirements include the use of the computation results on the subsequent steps and preselection of the candidate angles. Other important questions include the integrability of the information gain over the set of solutions (cfr. Eq. (2)), and the influence of the class of template images and the candidate angle sets on the performance of the proposed approach. These questions will be addressed in future work.

\section{Conclusion}

In this paper, an acquisition algorithm for dynamic angle selection in grey scale computed tomography was proposed. In this approach, the angle from which a new projection needs to be acquired to gain the most information about the object, is dynamically computed. Prior knowledge about the object itself is not required. Simulation experiments showed that this approach can forecast projection angles that lead to more accurate reconstructions from fewer projections compared to the widely used angle selection approaches. The proposed method is well suited in X- ray imaging scenarios where the acquisition of a single projection is expensive (in terms of acquisition time, dose, or object-source positioning). The highest gain is expected for objects with a small number of preferential directions in the shape and interior. Currently, the computation time of the proposed algorithm is very high for large experimental datasets. Future work will focus on improving the efficiency of the algorithm.

\section{Acknowledgments}

This work was financially supported by the BOF LP project 25778 and the SBO project TomFood from the Agency for Innovation by Science and Technology in Flanders (IWT). K.J.B. was supported by the Netherlands Organisation for Scientific Research (NWO), programme 639.072.005.

\section{Appendix A. Proof of the upper bound for the diameter of the solution set}

Let $\Theta=\left\{\theta_{1}, \ldots, \theta_{d}\right\}$ be the set of $d$ angles, for which projection data $p^{\Theta}=W^{\Theta} v$ of the unknown image $v \in[0,1]^{n}$ have already been measured, where $n$ is the number of pixels in the image, $W^{\Theta}=\left(w_{i j}^{\Theta}\right) \in \mathbb{R}^{m \times n}$ is the projection matrix corresponding to $\Theta$, and $m$ is the total number of measurements in the projection data $p^{\Theta} \in \mathbb{R}^{m}$. Let $S_{W^{\Theta}}\left(p^{\Theta}\right)=\left\{x \in[0,1]^{n}: W^{\Theta} x=p^{\Theta}\right\}$ be the set of all solutions that are consistent with the projection data $p^{\Theta}$.

Lemma 1. Let $x \in S_{W^{\Theta}}\left(p^{\Theta}\right)$. Then $\|x\|_{2}^{2} \leqslant \frac{\left\|p^{\Theta}\right\|_{1}}{d}$.

Proof of Lemma 1. For the strip projection model, $\sum_{i=1}^{m} w_{i j}^{\Theta}=d$ $(j=1, \ldots, n)$, as the total pixel weight for each projection angle is equal to the area of a pixel, which is 1 , and there are $d$ projection angles. Since $p_{i}^{\Theta} \geqslant 0(i=1, \ldots, m)$, we have $\left\|p^{\Theta}\right\|_{1}=\sum_{i=1}^{m} p_{i}^{\Theta}$ and

$\sum_{i=1}^{m} p_{i}^{\Theta}=\sum_{i=1}^{m}\left(\sum_{j=1}^{n} w_{i j}^{\Theta} x_{j}\right)=\sum_{j=1}^{n}\left(\sum_{i=1}^{m} w_{i j}^{\Theta}\right) x_{j}=\sum_{j=1}^{n} d x_{j}$

and hence $\left\|p^{\Theta}\right\|_{1}=d \sum_{j=1}^{n} x_{j}$.

As $x \in[0,1]^{n},\|x\|_{2}^{2} \leqslant\|x\|_{1}=\sum_{j=1}^{n} x_{j}=\frac{\left\|p^{\Theta}\right\|_{1}}{d}$.

Let $x^{*}$ be the shortest solution in $S_{W^{\Theta}}\left(p^{\Theta}\right)$, in the Euclidean sense. Define the central radius by $R=\sqrt{\frac{\left\|p^{\Theta}\right\|_{1}}{d}-\left\|x^{*}\right\|_{2}^{2}}$. The following theorem allows to find an upper bound for the Euclidean distance between two solutions from $S_{W^{\Theta}}\left(p^{\Theta}\right)$.

Theorem 1. Let $x, y \in S_{W^{\Theta}}\left(p^{\Theta}\right)$. Then $\|x-y\|_{2} \leq 2 R$.

Proof of Theorem 1. From the definition of $x^{*}$ we have $\left(x-x^{*}\right) \in \mathcal{N}\left(W^{\Theta}\right)$ and $x^{*} \perp\left(x-x^{*}\right)$. Using the Pythagoras' theorem and Lemma 1 yields

$\left\|x-x^{*}\right\|_{2}^{2}=\|x\|_{2}^{2}-\left\|x^{*}\right\|_{2}^{2} \leqslant \frac{\left\|p^{\Theta}\right\|_{1}}{d}-\left\|x^{*}\right\|_{2}^{2}=R^{2}$

Therefore,

$\|x-y\|_{2} \leqslant\left\|x-x^{*}\right\|_{2}+\left\|y-x^{*}\right\|_{2} \leq 2 R$.

\section{References}

[1] M.J. Murphy, J. Balter, S. Balter, J.A. BenComo Jr., I.J. Das, S.B. Jiang, C.-M. Ma, G.H. Olivera, R.F. Rodebaugh, K.J. Ruchala, H. Shirato, F.-F. Yin, The management of imaging dose during image-guided radiotherapy: report of the AAPM Task Group 75, Med. Phys. 34 (10) (2007) 4041-4063.

[2] P.A. Midgley, R.E. Dunin-Borkowski, Electron tomography and holography in materials science, Nat. Mater. 8 (4) (2009) 271-280. 
[3] L. Varga, P. Balazs, A. Nagy, Direction-dependency of binary tomographic reconstruction algorithms, Graph. Models 73 (2011) 365-375.

[4] Z. Zheng, K. Mueller, Identifying sets of favorable projections for few-view lowdose cone-beam CT scanning, in: 11th International Meeting on Fully ThreeDimensional Image Reconstruction in Radiology and Nuclear Medicine, Potsdam, Germany, 2011, pp. 314-317.

[5] G. Placidi, M. Alecci, A. Sotgiu, Theory of adaptive acquisition method for image reconstruction from projections and application to EPR imaging, J. Magn. Reson. Ser. B 108 (1) (1995) 50-57.

[6] M. Venere, H. Liao, A. Clausse, A genetic algorithm for adaptive tomography of elliptical objects, IEEE Signal Process. Lett. 7 (7) (2000) 176-178.

[7] K.J. Batenburg, W.J. Palenstijn, P. Balazs, J. Sijbers, Dynamic angle selection in binary tomography, Comput. Vision Image Understand. 117 (4) (2013) 306318.

[8] K.J. Batenburg, W. Fortes, L. Hajdu, R. Tijdeman, Bounds on the quality of reconstructed images in binary tomography, Discrete Appl. Math. 161 (15) (2013) 2236-2251
[9] W. Fortes, K.J. Batenburg, J. Sijbers, Practical error bounds for binary tomography, in: Proceedings of the First International Conference on Tomography of Materials and Structures, ICTMS 2013, 2013, pp. 97-100.

[10] Y. Saad, Iterative Methods for Sparse Linear Systems, 2nd Edition., Society for Industrial and Applied Mathematics, Philadelphia, PA, USA, 2003.

[11] J. Gregor, T. Benson, Computational analysis and improvement of SIRT, IEEE Trans. Med. Imag. 27 (7) (2008) 918-924.

[12] W.J. Palenstijn, K.J. Batenburg, J. Sijbers, The ASTRA tomography toolbox, in: 13th International Conference on Computational and Mathematical Methods in Science and Engineering, CMMSE 2013, 2013.

[13] W.J. Palenstijn, K.J. Batenburg, J. Sijbers, Performance improvements for iterative electron tomography reconstruction using graphics processing units (GPUs), J. Struct. Biol. 176 (2) (2011) 250-253.

[14] H. Guan, R. Gordon, Computed tomography using algebraic reconstruction techniques (ARTs) with different projection access schemes: a comparison study under practical situations, Phys. Med. Biol. 41 (9) (1996) 1727-1743. 